\title{
Body Surface Mapping of the Mechanical Cardiac Activity
}

\author{
Kim Munck, Bolette Dybkjær Hansen, Nina Jacobsen, Louise Pedersen Pilgaard, \\ Samuel Schmidt, Kasper Sørensen, Johannes Jan Struijk \\ Aalborg university, Aalborg, Denmark
}

\begin{abstract}
Introduction: Studies of the movement of the chest wall show their potential in the diagnosis of heart diseases. Few studies have focused on mapping these movements especially in the lower inaudible frequency band.

Aims: This study evaluates Body Surface Mapping $(B S M)$ as a method for describing mechanical cardiac activity.

Methods: The chest wall's velocity was measured with a Laser Doppler Vibrometer (LDV) on six healthy subjects. The measuring procedure was repeated for 30 points positioned in a grid at the subjects chest. An electrocardiogram $(E C G)$ and respiration was measured to support the signal processing. The heart movement were described using amplitude maps, constructed from the integrated LDV signal components of 1-20 Hz.

Results: The impact of the cardiac motion on the displacement of the chest wall was shown as a typical pattern of the changes of the amplitude maps as a function of time.

Conclusion: The results had a high reproducibility and were in concordance with existing evidence, thus indicating BSM to be a valid method for characterization of the mechanical cardiac activity.
\end{abstract}

\section{Introduction}

Acoustic measurements on the chest surface have shown a high potential in diagnosing cardiac diseases. This includes not only recordings of the high frequency signals, such as valvular sounds and murmurs, but also low frequency, subsonic, signals as obtained with, e.g., seismocardiography. [1]

BSM of heart sounds obtained from the chest has been investigated as an indicator for diagnosis of sleep apnea [2]. Acoustic BSM from the chest, also shows potential to detect the origin of heart sounds and murmurs [3]. However, BSM of the mechanical cardiac activity is still an unexplored area.

The aim of this study is to evaluate BSM, obtained with a LDV, as a method for exploring the mechanical cardiac activity.

\section{Method}

Seven Caucasian subjects were included: four males and three females. One male subject suffered from a known heart disease, and was excluded from further data analysis. Inclusion criterion was age within 18 to 70 years and exclusion criteria were implants or tattoos within the measurement area, abnormal murmurs or abnormal heart sounds. The average age was $31.3 \pm 11.7$ years and the average body mass index was $23.2 \pm 2.9 \mathrm{~kg} / \mathrm{m}^{2}$.

\subsection{Data collection}

The normal skin velocities were measured sequentially from 30 points on the subjects chest with an LDV (Polytec PDV-100). The measurement points were placed in a $6 \times 5$ grid. At each grid point 40 seconds of signal was obtained. Placements of the grid points are illustrated in Figure 1. The subjects lay in a supine position, relaxing for ten minutes before start of the recording. The subjects were asked to breath with approximately five second intervals between exhalation and inhalation during the recording period.

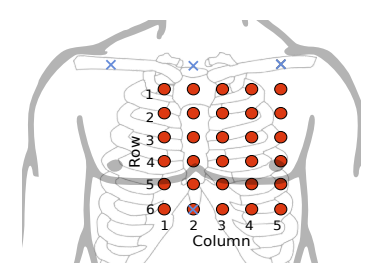

Figure 1. Measurement points (o) were adjusted according to the size of the subjects chest. Reference points $(\times)$ were positioned at the suprasternal notch, the coracoid processes and the xiphoid process. The mean grid length was $17.3 \pm 1.9 \mathrm{~cm}$ and the mean width was $17.4 \pm 1.9 \mathrm{~cm}$.

The LDV's 1,000 Hz lowpass filter was used and the range was set to measure velocities up to $20 \mathrm{~mm} / \mathrm{s}$. The LDV was positioned at an optimal distance, $23.4 \mathrm{~cm}$ above the measurement point. The ECG was obtained using the Mason-Likar placement of the limb leads and a respiration belt was used to detect the respiratory cycle. An accelerometer was mounted on the LDV to monitor any 
external vibrations. All four signals were sampled simultaneously by an iWorx RA-834 with a sample frequency at $10,000 \mathrm{~Hz}$. Measurements were discarded if the noise level, measured by the accelerometer, exceeded background noise level or if the subject moved or spoke. The experimental set up is illustrated in Figure 2.

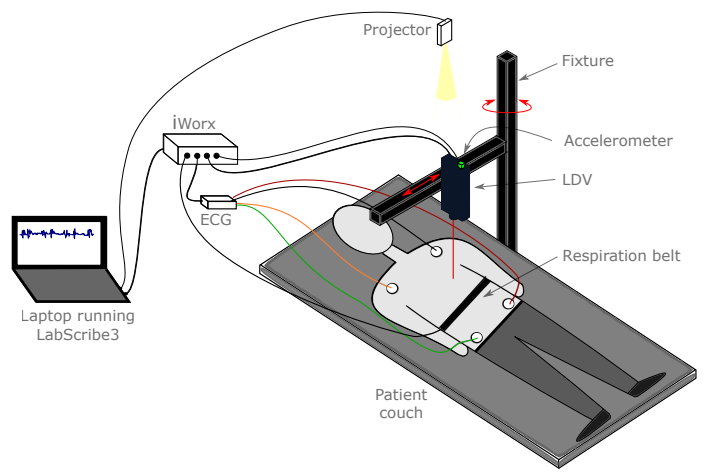

Figure 2. The experimental setup. The grid of measurement points were projected on the subjects chest. An iWorx RA-834 sampled the signals from the LDV, the ECG electrodes, the accelerometer and the respiration belt simultaneously. The LDV was mounted on a fixture, along with the accelerometer.

\section{Signal processing}

Along with the LDV measurements, ECG and respiration signals were utilized to support the segmentation of the signal into separate heart beats with reference to the respiration phase. Lead I of the ECG signal was filtered with a $50 \mathrm{~Hz}$ IRR comb filter and normalized according to the method described by Zhong et al. [4]. R-peaks were detected using maxima detection above a threshold at $25 \%$ of the normalized signal. The respiration signal was normalized and a threshold was used to determine the respiratory phase to capture the periods between exhalations and inhalations.

\subsection{Preprocessing}

Due to dropouts in the LDV signal, dropout removal was necessary. Approximately 20 dropouts were observed per second, each with a typical length of $4 \mathrm{~ms}$ and with the characteristics of an impulse. All dropouts were identified by their high frequency contents, and replaced by $1^{\text {st }}$ order interpolations of the signal. The LDV output is the velocity of the chest wall. To derive the chest wall displacement, the LDV signal was integrated, as illustrated in Figure 3.

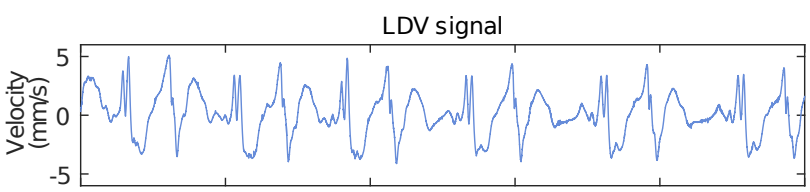

Integrated LDV signal
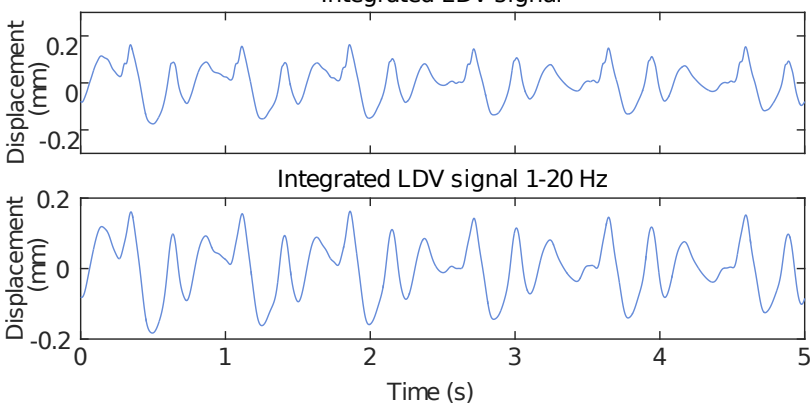

Figure 3. From top to bottom: LDV signal, integrated LDV signal, and the integrated LDV signal in the frequency band $1-20 \mathrm{~Hz}$. The illustrated signal was measured at row 3 and column 3 in the grid.

\subsection{Estimation of a mean heart beat}

A $1-20 \mathrm{~Hz}$ bandpass FIR filter of $1024^{\text {th }}$ order was applied to extract information about the heart movement. The pass band was inspired by Wick et al. [5].

With the purpose of extracting the cardiac cycles from the filtered LDV signal, the signals were segmented. The segmentation was performed using synchronized information from both the ECG and the respiration signal. ECG recordings were used since the onset of the cardiac cycle can be determined in relation to the R-peak [6]. The second heart sound indicated the end of the systole [6] and the end of the segments.

The segments describing the cardiac cycles' displacement were collected, cut to in accordance with the systole and averaged using cross-correlation to create the mean systolic beat as illustrated in Figure 4. [7]

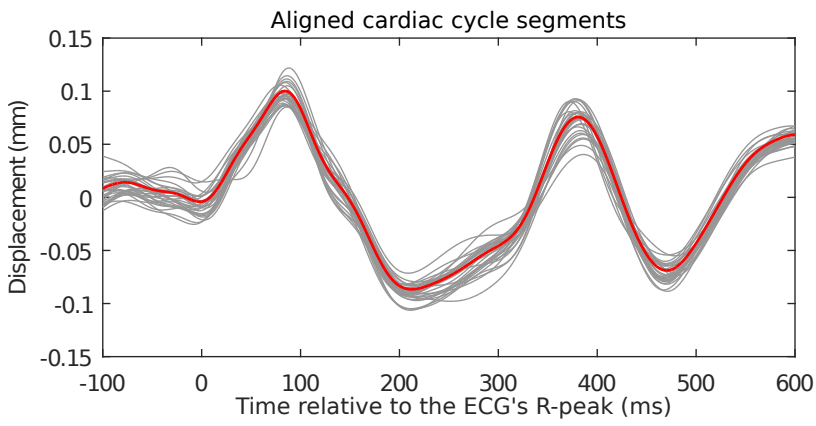

Figure 4. Aligned cardiac segments (gray) and their mean (red). The illustrated segments were measured at row 3 and column 3 in the grid. 
The mean systolic beat, for each of the grid points, were combined into a series of amplitude maps, revealing the spatial displacement as a function of time. Amplitude maps for every $20 \mathrm{~ms}$, from $20 \mathrm{~ms}$ before the R-peak until $20 \mathrm{~ms}$ after the onset of S2 was generated and analysed. Amplitude maps were constructed for all subjects, describing positive, negative and neutral displacement of the chest wall. A positive displacement corresponds to an anterior movement, whereas a negative displacement corresponds to a posterior movement of the chest wall. Amplitude maps for one subject are illustrated in Figure 5.

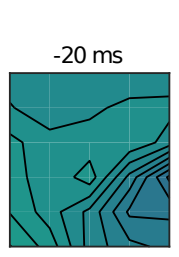

$60 \mathrm{~ms}$

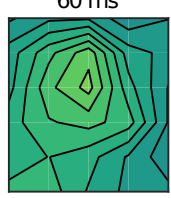

$140 \mathrm{~ms}$

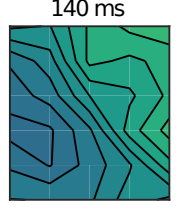

$220 \mathrm{~ms}$

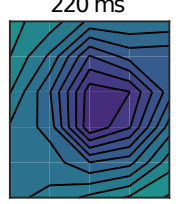

$300 \mathrm{~ms}$

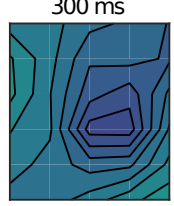

$-0.1$

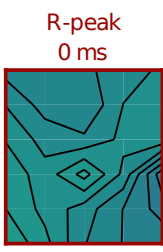

$80 \mathrm{~ms}$

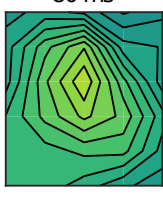

$160 \mathrm{~ms}$

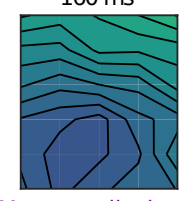

Max neg displacement $240 \mathrm{~ms}$

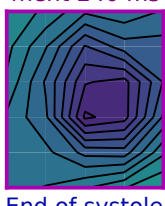

End of systole

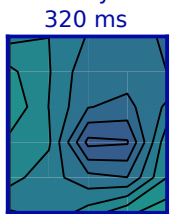

Displacement $(\mathrm{mm})$

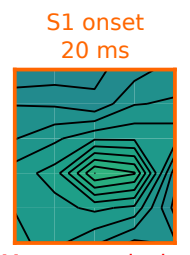

Max pos velocity Max pos displace$100 \mathrm{~ms}$

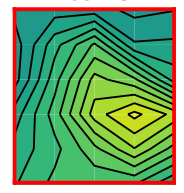

$180 \mathrm{~ms}$

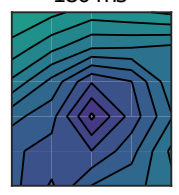

$260 \mathrm{~ms}$

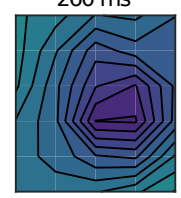

$340 \mathrm{~ms}$

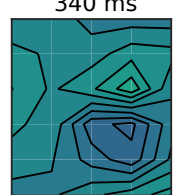

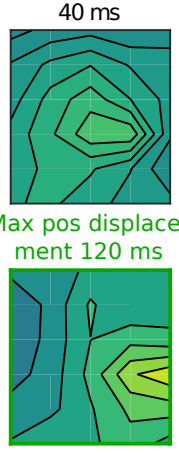

$200 \mathrm{~ms}$

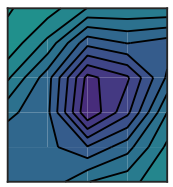

$280 \mathrm{~ms}$

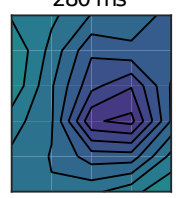

$360 \mathrm{~ms}$

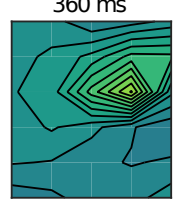

0.1
Figure 5. Amplitude map for one subject. Each amplitude map illustrates the positive, anterior (yellow) and negative, posterior (purple) displacement of the chest wall. Each amplitude map was constructed using signals from all 30 measuring points. The maps cover the systole.

\section{4. $\quad$ Results}

Each amplitude map covers all 30 measurement points. Three general tendencies were observed for all subjects, as described in table 1 .

Table 1. Extracted features present in all subjects including time and location of occurrences. Times are relative to the onset of the S1 heart sound. The transverse position is relative to the sternum where left is positive and the longitudinal position is relative to the suprasternal notch where the inferior direction is positive. Values are written as mean \pm std.

\begin{tabular}{lccc}
\hline \multirow{2}{*}{ Event } & Time & \multicolumn{2}{c}{ Position (mm) } \\
& $(\mathrm{ms})$ & Transverse & Longitudinal \\
\hline $\begin{array}{l}\text { Maximum } \\
\text { positive } \\
\text { velocity }\end{array}$ & $50 \pm 40$ & $-2.9 \pm 30$ & $98 \pm 95$ \\
$\begin{array}{l}\text { Maximum } \\
\text { positive } \\
\text { displacement }\end{array}$ & $68 \pm 46$ & $50 \pm 17$ & $180 \pm 68$ \\
$\begin{array}{l}\text { Maximum } \\
\text { negative } \\
\text { displacement }\end{array}$ & $160 \pm 61$ & $64 \pm 32$ & $135 \pm 56$ \\
\hline
\end{tabular}

In each subject two opposite displacement were observed during the systole. The first, a positive displacement, which becomes dipolar with a predominantly horizontal orientation, and subsequently a negative maximum displacement with the impression of a more longitudinal dipolar pattern. An example is shown in Figure 5 at 120 $\mathrm{ms}$ to $160 \mathrm{~ms}$.

\section{Discussion}

Maximum positive chest wall displacement, maximum negative chest wall displacement, and maximum chest wall velocity were extracted from the amplitude maps.

The timing of the maximum velocity, measured between the R-peak and the maximum positive chest wall displacement, was at $50 \pm 40 \mathrm{~ms}$ after the onset of S1. The closure of the aortic and pulmonic valves occur $47 \pm 7 \mathrm{~ms}$ after the onset of the systole, indicating the end of the isovolumetric contraction, according to Luo et al. [8]. The results from this study agree with O'Rourke et al. [9], who state that an anterior movement of the left ventricle happens with the onset of the isovolumetric contraction. The maximum anterior movement occurs simultaneous with, or just after the opening of the aortic valve [9]. Luo et al. [8] found the isovolumetric contraction time for patients with HF to be $70.2 \pm 14.6 \mathrm{~ms}$, which is longer than the isovolumetric contraction time for healthy people. For this reason, the maximum positive velocity for patients with HF might be later as well. 
The isovolumetric contraction is followed by ventricular ejection. After the initial rapid ejection, the left ventricular wall retracts from the chest wall and well before S2, the apex retracts and returns to baseline. [9] The end of rapid ejection in this study, might coincide with the maximum positive chest wall displacement occurring at $68 \pm 46 \mathrm{~ms}$ after the onset of $\mathrm{S} 1$. The left ventricular retraction appears at the time of the maximum negative displacement, which was found to be $160 \pm 61 \mathrm{~ms}$.

After the maximum positive displacement a negative displacement near the sternum were observed at the same time as a positive displacement was observed at the left side of the chest. The negative displacement area then expands, and the positive displacement area moves upwards. This pattern might indicate a movement of the heart which could indicate a rotation, as described by O'Rourke et al. [9] and Luo et al. [8].

A reproducibility test revealed a high correlation between the signals of two different days, indicating a high reproducibility of the method.

To evaluate BSM as a method for characterisation of mechanical cardiac activity, further studies with a larger number of subjects are necessary. Further characterisation and signal processing of the obtained signals and maps could bring additional insight to the potential of the method. To validate BSM as a method for diagnosis of cardiac disease studies including both healthy subjects and subjects with such diseases are necessary. Substituting the LDV with accelerometers might give more information to the BSM.

\section{Conclusion}

Surface mapping of chest displacement using a Laser Doppler Vibrometer is a promising method to study cardiac movement and events in the cardiac cycle. A further study of this method in relation to cardiac disease may add an interesting new modality for the diagnosis and monitoring of cardiac disease in the clinic.

\section{References}

[1] Hu Y, Kim EG, Cao G, Liu S, Xu Y. Physiological acoustic sensing based on accelerometers: a survey for mobile health- care. Ann Biomed Eng Nov 2014;42(11):2264-2277.

[2] Rendon DB, Rojas Ojeda JL, Crespo Foix LF, Morillo DS, Fernandez MA. Mapping the human body for vibrations using an accelerometer. Conf Proc IEEE Eng Med Biol Soc 2007;2007:1671-1674.

[3] Cozic M, Durand LG, Guardo R. Development of a cardiac acoustic mapping system. Med Biol Eng Comput Jul 1998; 36(4):431-437.

[4] Zhong L, Guo X, Ji A, Ding X. A Robust Envelope Extraction Algorithm for Cardiac Sound Signal Segmentation. Institute of Electrical \& Electronics Engineers (IEEE), may 2011.

[5] Wick CA, Inan OT, McClellan JH, Tridandapani S. Seismocardiography-based detection of cardiac quiescence. IEEE Transactions on Biomedical Engineering August 2015; 62(8):2025-2032.

[6] Seeley R, VanPutte C, Regan J, Russo A. Seeley's Anatomy and Physiology. McGraw-Hill, 2011.

[7] Rangayyan RM. Biomedical Signal Analysis (IEEE Press Series on Biomedical Engineering). Wiley-IEEE Press, 2015.

[8] Luo X, Cao T, Li Z, Duan Y. A preliminary study on the evaluation of relationship between left ventricular torsion and cardiac cycle phase by two-dimentional ultrasound speckle tracking imaging. Int J Cardiovase Imaging May 2009; 25(6):559-568.

[9] O'Rourke RA, Shaver JA, Silverman ME. Hurst's The Heart. The McGraw-Hill Companies, 2008; 215-293.

\section{Appendix}

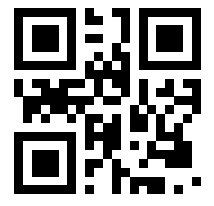
Figure 6. The QR-code links to a video representation of the amplitude maps for one cardiac systolic cycle from one subject. The video is slowed down 100 times and is running in 30 seconds.
Address for correspondence:

Kim Munck Jeppesen

Fredrik Bajers Vej 7,

9220 Aalborg Ø, DK.

kkj@hst.aau.dk 